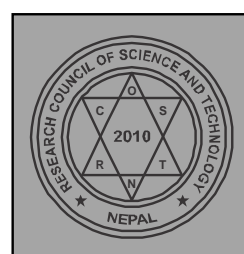

\title{
BIBECHANA
}

A Multidisciplinary Journal of Science, Technology and Mathematics

ISSN 2091-0762 (online)

Journal homepage: http://nepjol.info/index.php/BIBECHANA

\section{Relativity in cosmologically preferred frame and the UHECR paradox}

\author{
Saroj Nepal \\ Post Graduate Department of Physics \\ M. M. A. M. Campus, Biratnagar, Nepal \\ Email: saroj.paradox@gmail.com \\ Accepted for publication: February 02, 2014
}

\begin{abstract}
The Greisen-Zatsepin-Kuzmin $(\mathrm{GZK})$ cutoff $\left(5 \times 10^{19} \mathrm{eV}\right)$ of special relativity in the observed ultra high energy cosmic rays (UHECR) spectrum is one of the most puzzling paradoxes in physics. Experimentally a number of cosmic ray events have been detected above this GZK limit which is known as UHECR paradox. We propose a resolution of this paradox through a modification of the relativistic kinematics keeping in mind that it should not lead to predictions different from those of special relativity in the well tested domains. It is shown that theoretical limit in UHECR spectrum can be explained in the framework of Lorentz invariance violation (LIV) theories which assume the existence of a preferred frame. The present paper proposes that the velocity of the solar system with respect to the rest frame of the universe plays a role in explaining the paradox.
\end{abstract}

(C) 2014 RCOST: All rights reserved.

\section{Keywords: Special Relativity (SR); GZK limit; Lorentz invariance violation; Lorentz transformation; Einstein synchrony.}

\section{Introduction}

The possibility that Lorentz invariance can be violated in nature has currently become a subject of interest. People often doubt if the special relativity (SR) is only an approximate symmetry of nature $[1,2]$. To give a quantitative measure of Lorentz invariance violation (LIV), one can build up a test theory where the Lagrangian of electrodynamics can be slightly deformed by adding to it a tiny Lorentz violating term. One such deformation considered by the authors of Ref. [1] (see also [3]) following standard practice causes the speed of light $c$ to differ from the maximum attainable speed $c_{0}$ (which hereafter, unless stated otherwise, will be assumed to be equal to 1) by a small velocity parameter $\varepsilon$ of the theory. The obvious consequence of this consideration is the existence of a preferred inertial frame of reference.

It is a common practice and also reasonable to assume this preferred frame to be "the rest frame of the universe" $\left(\Sigma_{0}\right)$ with respect to which the cosmic microwave background radiation (CMBR) is isotropic. Let us call it the rest frame of the cosmic substratum (RFCS).

Precision tests for anisotropies in velocity of light due to the motion of the solar system relative to the CMBR frame have set a limit on this $\varepsilon[1,4]$,

$$
|1-c|=|\varepsilon|<3 \times 10^{-22} .
$$


However it has been argued [1-3] that stronger constraints on $\varepsilon$ can be obtained, not from precision tests, but from observations on ultra high energy cosmic rays (UHECR). For example, if $c<1$ it has been shown that the mere detection of primary proton energy up to $100 \mathrm{EeV}$ set the bound on $\varepsilon$ more than one order of magnitude stronger:

$$
|\varepsilon|<5 \times 10^{-24} \text {. }
$$

The physical basis for obtaining such a bound is that a particle can be super luminal in vacuum (if $c<1$ ) in which case, a proton being a charged particle will in its passage, quickly lose energy through the so called "vacuum Cerenkov radiation" and will therefore fail to be detected with the super luminal speed. The last bound on $|\varepsilon|$ is obtained by equating the speed of proton at $100 \mathrm{EeV}$ with the speed of light c, then subtracting it from unity, the latter being the limiting speed of SR. The limit on $\varepsilon$ thus obtained does not require (unlike the way it is obtained through precision test mentioned before) any assumption regarding the motion of the laboratory frame with respect to $\Sigma_{0}$. LIV is also much discussed in connection with one of the most puzzling paradoxes in physics concerning UHECRs. One quite robust predictions of special relativity is the existence of the so called Greisen-Zatsepin-Kuzmin (GZK) phenomenon, which tells us that cosmic ray protons coming from cosmological distances with energies above certain limiting value (GZK cutoff), should not be observed on Earth. The predicted value for this catastrophic cutoff is $5 \times 10^{19} \mathrm{eV}$. This value corresponds to the threshold energy for photo-pion production by cosmic ray protons interacting with soft CMBR photons which pervade the universe. However some recent experiments have shown that this relativistically calculated threshold energy seems to be too low. Indeed recently ground based detectors have detected over about a hundred events near and above the GZK cutoff and a double digit number of events with energies at or above $10^{20} \mathrm{eV}$. The highest energy cosmic ray so far has been the $3.2 \times 10^{20} \mathrm{eV}$ detected by the Fly's Eye air shower detector in Utah [5]. However if the sources of UHECRs are really extragalactic (there are ample reasons to believe so[6]) and since the calculation of GZK limit is so robust that even one event at $10^{20} \mathrm{eV}$ "appears surprising" [7]. The arrival of UHECR on Earth with energies above the GZK threshold is known as the UHECR paradox [8-10] mentioned in the beginning of this paragraph. There have been exotic proposals in the literature which try to explain the trans GZK cosmic ray events in the framework of LIV theories which assume the existence of a preferred frame $[2,9,11]$. Let us call them preferred frame theories. As an example, according to one most popular scenarios [12], existence of different maximal speeds for different particle species is assumed and they are also assumed in general to differ from the speed of light in vacuo [see Ref.[2] and references therein]. In this way, introduction of small LIV has been shown to have effects that increase rapidly with energy in such a manner that ultimately inelastic collisions with CMBR photons become kinematically forbidden [2].

The present paper proposes that the velocity of the solar system with respect to the rest frame of the universe might play a role in explaining the paradox.

In an effort to look for new physics, when one considers theories involving LIV one still believes that behavior of moving rods and clocks is still governed by the Lorentz transformation (LT). However, other laws of physics might not strictly remain covariant under LT. For example one may consider the possibility that causal cone need not coincide with the light cone [13], i.e the speed of light may not be the same as the invariant speed $c$ of LT.

However if one is prepared to do away with the principle of relativity, or in other words if one believes in the existence of a preferred inertial frame, there is no point in holding on to the belief that standard rods and clocks of different inertial frames behave strictly according to LT. Note that after all LT is a consequence of the relativity principle. Hence in search for a new physics one may consider the possibility of a deformed LT (not just a deformed dispersion relation) to relate observations performed by different inertial observers. Once such a transformation is guessed, other aspects of kinematics such as expressions for momentum $\mathbf{p}$ and energy $\mathrm{E}$ of a particle or the dispersion relation can be obtained through 
a kind of 4-vector formulation (see below). Clearly the predictions of the deformed LT will be different from those of the relativity theory. However the difference in the predictions must be undetectable in the domain where special relativity has been tested beyond doubt.

In the following we shall look for such a transformation that will be capable to explain the UHECR paradox and at the same time will be able to reproduce the standard relativistic results. We know that Einstein obtained his transformations deductively from his relativity and the "constancy of velocity of light" (CVL) postulates. If the relativity postulate is sacrificed what guidelines should one follow in order to guess the transformation equation? The next section will provide an answer to this question.

\section{Transformation Equations}

Although the kinematics of relativity theory was obtained by Einstein from a general principle like the relativity of motion and a principle concerning the speed of light, the operative aspects of these postulates used in the derivation can be laid down in more concrete terms. Indeed if one consults a standard text book on relativity, one finds that the derivation of LT starts from the assumption of a linear transformation with unknown coefficients which are determined using essentially the following operative inputs:

(1) The coordinate clocks in any inertial frame are assumed to be synchronized by light signal following the Einstein synchrony or the standard synchrony, according to which the one-way-speed (OWS) of light is assumed to be the same as its two-way-speed (TWS) in any direction [14-16].

(2) The speed of light is the (i) same and (ii) isotropic with respect to all inertial observers.

(3) Measuring rods placed perpendicular to its direction of motion do not undergo any contraction or elongation with respect to its rest length.

The first of the above is just a synchronization convention but the other two items are the consequences of the relativity principle. A little amplification of this statement in respect of item (2) may be in order. One might think that (2) is equivalent to Einstein's CVL postulate. This is indeed a misconception [17]. The CVL postulate of Einstein refers to constancy with respect to change in the velocity of light source. In effect this postulate emphasizes the wave character of light. Once wave is launched it is no longer linked to the source. Indeed Einstein's second postulate concerning the speed of light in conjunction with the principle of relativity only imply the constancy with respect to the change of the inertial observer as well [17]. In a preferred frame theory where the principle of relativity is expected to be violated, the transformation equations cannot be obtained with item (2) as an input which, as explained, depends on the relativity principle although CVL can be used in the stationary frame. As regards input (1) however there is no difficulty but there is no special advantage in synchronizing coordinate clocks using light signal. One may then ask what if the clocks were synchronized by some other signal say an "acoustic signal" for example. One may consider a substratum which can support such a signal and through which different inertial frames are supposed to move. To effect the synchronization, like the standard synchrony we shall stipulate the OWS of the signal along a straight line be equal to its TWS along the line in any frame $\Sigma_{\mathrm{k}}$. It has been shown elsewhere[14] that if input (2) is withheld, and the coordinate clocks of any inertial frame is synchronized by "acoustic signal", the transformation equation between a preferred frame $\Sigma_{0}$ and an arbitrary inertial frame $\Sigma_{\mathrm{k}}$ can be obtained as,

$$
\begin{aligned}
& x_{k}=\left(a_{k x} / a_{k y}\right)\left(1-u_{0 k}^{2} / a_{0}^{2}\right)^{-1 / 2}\left(x_{0}-u_{0 k} t_{0}\right), \\
& t_{k}=\left(a_{0} / a_{k y}\right)\left(1-u_{0 k}^{2} / a_{0}^{2}\right)^{-1 / 2}\left(t_{0}-u_{0 k} x_{0} / a_{0}^{2}\right),
\end{aligned}
$$

where $\left(x_{0}, t_{0}\right)$ and $\left(x_{k}, t_{k}\right)$ refer to space-time coordinates as measured with respect to the stationary $\left(\Sigma_{0}\right)$ and moving frame $\left(\Sigma_{\mathrm{k}}\right)$ respectively. The relative velocity of $\Sigma_{k}$ with respect to $\Sigma_{0}$ has been denoted by $u_{0 k}$. As regards other terms, $a_{0}$ denotes the isotropic "acoustic speed" (two way or one way) in the stationary 
substratum, whereas $a_{k x}$ and $a_{k y}$ are the TWS' of the synchronizing signal in $\Sigma_{k}$ parallel (along the $x$ direction) and perpendicular (along the $y$-direction) to its direction of motion respectively. Note that in general $a_{k x}$ and $a_{k y}$ are expected to be functions of $u_{0 k}$ and hence the above equations are only formal and not usable unless some phenomenological assumptions are made regarding these functions. For optical signal synchronization we replace the terms $a_{k x}, a_{k y}$ and $a_{0}$ in Eqs.(3) and (4) by $c_{k x}, c_{k y}$ and $c_{0}$ respectively where the latter three terms represent the respective speeds of the light signal. In the relativistic world, by input (2), one finds in any $\Sigma_{k}$

$$
c_{k x}\left(u_{0 k}\right)=c_{k y}\left(u_{0 k}\right)=c_{0},
$$

and the above equations (Eqs.(3) and (4)) turns out to be LT under optical synchronization. We now ask what if Eq.(5) is approximately valid, so that the speed of light is almost and not quite independent of the speed of the reference frame with respect to a "preferred" one. Note that the transformation equations (3) and (4) are now most appropriate to deal with such questions. We now wish to use input (2) in these equations by modifying the former minimally. We try this by preserving the isotropy component (2(ii)) and relaxing the constancy component (2(i)) of the said input. Thus TWS of light is assumed to be isotropic in any frame $\Sigma_{k}$ and now we conjecture that this isotropic speed depends on $u_{0 k}$ in following way,

$$
c_{k x}=c_{k y}=c_{k}=c_{0}\left(1+\alpha u_{0 k}^{2} / c_{0}^{2}\right)^{1 / 2},
$$

where we have introduced a dimensionless constant $\alpha$ which is assumed to have such a small value that the proposed theory does not differ in its predictions from that so far tested relativistically. Clearly Eq.(5) is now replaced by Eq.(6) which approximately reduces to Eq.(5) for $\alpha u_{0 k}{ }^{2} / c_{0}{ }^{2} \ll<1$. Note that, depending on the smallness of $\alpha, u_{0 k}$ can be very close to $c_{0}$ and yet the last condition can still remain valid. We shall show below that if the phenomenological assumption described by Eq.(6) is believed to be true, the UHECR paradox can be explained in terms of the motion of the solar system with respect to the RFCS. We conclude this section by quoting the relevant transformation equations which are obtained by plugging in Eq.(6) in Eqs.(3) and (4):

$$
\begin{aligned}
& x_{k}=\left(1-u_{0 k}{ }^{2} / c_{0}{ }^{2}\right)^{-1 / 2}\left(x_{0}-u_{0 k} t_{0}\right), \\
& t_{k}=\left(1+\alpha u_{0 k}{ }^{2} / c_{0}{ }^{2}\right)^{-1 / 2}\left(1-u_{0 k}{ }^{2} / c_{0}{ }^{2}\right)^{-1 / 2}\left(t_{0}-u_{0 k} x_{0} / c_{0}{ }^{2}\right) .
\end{aligned}
$$

\section{Metric and 4-Vectors}

In SR classical expressions for momentum and energy had to be altered in order for the conservation principles to be Lorentz covariant. These expressions can easily be obtained by writing the energy momentum conservation in terms of a 4-vector relation. The energy momentum 4-vectors are obtained in terms of the invariant interval of SR. In the present situation, such a thing cannot be obtained easily since one recalls that the notion of invariant interval of SR is an outcome of the existence of an invariant speed $c\left(c_{0}\right)$ of the theory. In the present context in absence of such a speed the invariant interval does not exist in the way it existed in SR. Besides, since there should exist a preferred frame, in order to obtain the correct conservation principle (or to obtain definition of energy and momentum) an appeal to covariance of physical laws cannot be made. In the following we suggest a way out. From the transformations (3) and (4) together with

$$
y_{k}=y_{0}, z_{k}=z_{0}
$$

It is evident that

$$
\left(c_{k y} / c_{k x}\right)^{2} x_{k}^{2}+y_{k}^{2}+z_{k}^{2}-c_{k y}^{2} t_{k}^{2}=x_{0}^{2}+y_{0}^{2}+z_{0}^{2}-c_{0}^{2} t_{0}^{2} .
$$

Recalling Eq. (6) the above relation reads

$$
x_{k}^{2}+y_{k}^{2}+z_{k}^{2}-c_{k}^{2} t_{k}^{2}=x_{0}^{2}+y_{0}^{2}+z_{0}^{2}-c_{0}^{2} t_{0}^{2},
$$

and in terms of the differential intervals one obtains the following invariant interval 


$$
d \tau^{2}=d t_{k}^{2}-\left(1 / c_{k}^{2}\right)\left(d x_{k}^{2}+d y_{k}^{2}+d z_{k}^{2}\right)
$$

and by analogy with SR we call $d \tau$ as the proper time interval.

Note that the expression for the above invariant interval is frame dependent unlike the case in SR because of the presence of $c_{k}\left(u_{0 k}\right)$ in the last expression. However one can easily develop a 4-vector formulation like that in SR by defining the 4-momentum of a particle of mass $\mathrm{m}$ as

$$
P=\left(m \gamma_{k}, m \gamma_{k}\left(v_{k}\right)_{i}\right)
$$

with

$$
\gamma_{k}=\left(1-v_{k}^{2} / c_{k}^{2}\right)^{-1 / 2},
$$

where $\left(v_{k}\right)_{i}$ represents the ith component of the three velocity $v_{k}$ of the particle in $\Sigma_{k}$. Imposing

where

$$
P . P=P^{2}=\eta_{\mu \nu} p_{\mu} p_{v}=\text { invariant }
$$

$$
\eta_{\mu v}=\left(1,-1 / c_{k}^{2},-1 / c_{k}^{2},-1 / c_{k}^{2}\right),
$$

one obtain the dispersion relation for the particle in any frame $\Sigma_{k}$ as

where

$$
E_{k}^{2}=p_{k}^{2} c_{k}^{2}+m^{2} c_{k}^{4}
$$

and

$$
p_{k}=m v_{k} /\left(1-v_{k}^{2} / c_{k}^{2}\right)^{1 / 2}=m \gamma_{k} v_{k}
$$

$$
E_{k}=m \gamma_{k} c_{k}^{2} .
$$

Although Eqs.(17), (18) and (19) look like the corresponding equations in SR, they are different since the relations are dependent on the frame considered, since now $c_{k}=c_{k}\left(u_{0 k}\right)$.

Note that expressions for energy, momentum and the dispersion relation reduce to the usual relativistic ones in the preferred frame $\Sigma_{0}$.

\section{Velocity Transformations}

Our theory therefore does not predict outcomes which are different from those in SR in $\Sigma_{0}$. The question now arises as to whether it is possible to predict a result significantly different from that of SR in a frame of reference (solar system) which is moving with a non-relativistic speed $\quad\left(u_{0 k} \approx 10^{-3}\right)$, with respect to RFCS $\left(\Sigma_{0}\right)$. The answer seems to be affirmative and we suspect that the resolution of the cosmic ray paradox lies in such a non-preferred frame effect of the theory. To understand this question let us first quote the velocity transformation laws that follow from the transformation relations. We first consider a particle (say a proton) travelling along the $x$-direction with speed $v_{0}$ with respect to $\Sigma_{0}$. The corresponding speed in $\Sigma_{k}$ will be obtained from the transformations (7) and (8) as

$$
v_{k}=\left(1+\alpha u_{k}^{2} / c_{0}^{2}\right)^{1 / 2}\left(v_{0} \pm u_{k}\right) /\left(1 \pm v_{0} u_{k} / c_{0}^{2}\right),
$$

where we have put $u_{k}$ for $u_{0 k}$ for brevity. We shall consider the speeds of the cosmic ray protons in $\Sigma_{0}$ to be very close to unity,

$$
v_{0}=1-\varepsilon_{0}
$$

where $\varepsilon_{0}$ is of the order of $10^{-22}$ (see below). With this range of values for $v_{0}$ and recalling $u_{k} \approx 10^{-3}$, the velocity transformation formula (Eq.(20)) can be approximated as

$$
v_{k}=\left(1+\alpha u_{k}^{2}\right)^{1 / 2} v_{0}
$$


where the terms of the order of $\varepsilon_{0}^{2}$ and $\varepsilon_{0} u_{k}$ have been neglected in comparison to unity. Although in obtaining Eq.(22) we have assumed the motion of the particles to be along the $x$-direction, interestingly it can be shown that the above relation holds even for particle travelling along any direction under the above mentioned approximation.

\section{Velocity Threshold and the Resolution of the Paradox}

Using the usual relativistic energy formula valid in $\Sigma_{0}$

$$
E_{0}=m /\left(1-v_{0}^{2}\right)^{1 / 2},
$$

the velocity threshold for proton in $\Sigma_{0}$ corresponding to the GZK threshold energy

$E_{0 t h}=5 \times 10^{19} \mathrm{eV}$ speed can be calculated as

$$
v_{0 t h}=1-1.76 \times 10^{-22} \text {. }
$$

Now we will provide a possible explanation for the apparent detection of the trans-GZK events in terms of the motion of the solar system with respect to the CMBR frame. A surprisingly small value of the parameter $\alpha$ of the theory will be found to do this job. In order to demonstrate this we first anticipate (see below) this value for $\alpha$ :

$$
\alpha=3.42 \times 10^{-16} \text {. }
$$

From Eq.(6) the speed of light in the laboratory frame $\Sigma_{k}$ (for which $u_{k} \approx 10^{-3}$ ) can approximately be written as

$$
c_{k}=1+\eta_{k} \approx\left(1+\alpha u_{k}^{2} / 2\right)
$$

where $\eta_{k}$ measures the departure of the light speed value in $\Sigma_{k}$ from unity. Clearly

$$
\eta_{k} \approx \alpha u_{k}^{2} / 2=1.71 \times 10^{-22} \text {. }
$$

However this term is absent in the preferred frame and as we have seen, the special relativistic results (formulas for energy, momentum, dispersion relation etc) hold in $\Sigma_{0}$ and hence GZK cut off value for proton energy obtained from SR is still valid in the CMBR frame. We shall see how this threshold value may appear to be about $3 \times 10^{20} \mathrm{eV}$ in $\Sigma_{k}$ as detected by Fly's eye air shower detector. Without going into the details of the experimental analysis we now speculate that the observed energy of a cosmic ray particle is its relativistic energy. We denote it by $E_{k}^{r e l}$ which is given by,

$$
E_{k}^{r e l}=m c_{0}^{2} /\left(1-v_{k}^{2} / c_{0}^{2}\right)^{1 / 2},
$$

where we have explicitly retained $c_{0}$ for clarity. Returning to the energy formula for a particle in our frame $\Sigma_{k}$ one notes that its value in the solar system (laboratory) practically does not differ from its relativistic value in $\Sigma_{0}$, as

$$
E_{k}=m c_{k}^{2} /\left(1-v_{k}^{2} / c_{k}^{2}\right)^{1 / 2} \approx m c_{0}^{2} /\left(1-v_{0}^{2} / c_{0}^{2}\right)^{1 / 2}=E_{0},
$$

where we have used

$$
v_{k}^{2} / c_{k}^{2}=v_{0}^{2} / c_{0}^{2},
$$

that follows from Eqs. (6) and (22).We have also assumed in Eq.(29), $c_{k}^{2} \approx c_{0}^{2}$, since the error involved in such an approximation is only about 1 part in $10^{22}$, which can be disregarded since ultimately we will have to explain a discrepancy much bigger than this error $\left(3 \times 10^{20} \mathrm{eV}\right.$ against $\left.5 \times 10^{19} \mathrm{eV}\right)$. The above energy formula (Eq. (29)) can also be expressed as

$$
E_{k}=m c_{k}^{2} /\left[1-v_{k}^{2} / c_{0}^{2}\left(1+\alpha u_{k}^{2} / c_{0}^{2}\right)\right]^{1 / 2} \approx m /\left[\left(1-v_{k}^{2}\right)^{1 / 2}\left(1+\alpha u_{k}^{2} / 2 \varepsilon_{k}\right)^{1 / 2}\right],
$$

where in arriving at the last approximate expression we have put $c_{0}=1$ again and defined,

$$
\varepsilon_{k}=1-v_{k} .
$$


Using Eq.(28), one obtains from Eq.(31)

$$
E_{k}^{r e l}=E_{k}\left(1+\alpha u_{k}^{2} / 2 \varepsilon_{k}\right)^{1 / 2}
$$

which by Eq.(6.29) can be written as

$$
E_{k}^{r e l} \approx E_{0}\left(1+\alpha u_{k}^{2} / 2 \varepsilon_{k}\right)^{1 / 2} .
$$

Note that this is the relativistic energy of a particle moving with speed $v_{k}$. We now calculate this relativistic value of energy for a proton having the GZK threshold energy $E_{\text {Oth }}$. Using the transformation (22) and assuming $v_{0}=v_{o t h}$, where the later is given by Eq.(24), one obtains the corresponding $v_{k}$ as

$$
v_{k}=1-\varepsilon_{k}=1-5 \times 10^{-24},
$$

giving

$$
\varepsilon_{k}=5 \times 10^{-24} .
$$

Using this value for $\varepsilon_{k}$ and assumed value for $\alpha$ (Eq.(25)) and finally putting $E_{0}=E_{0 t h}$, we find from Eq.(34)

$$
E_{k}^{r e l} \approx 3 \times 10^{20} \mathrm{eV},
$$

which is nothing but the energy of the $300 \mathrm{EeV}$ event detected by the Fly's Eye. Therefore we conclude that the value of the parameter $\alpha \approx 3.42 \times 10^{-16}$ can explain the apparent detection of trans-GZK events. Note that the above calculation (or the choice of the value for $\alpha$ ) depends on the assumption that the 300 $\mathrm{E} e \mathrm{~V}$ event corresponds to the cut off value. However, it may not be so, indeed in future, a bit higher energy event may be detected, in which case the value of $\alpha$ will slightly go up. But this will not pose much problem since the assumed value of $\alpha$ is so small, it has enough flexibility to increase even substantially without contradicting SR in the tested domain.

\section{Discussions}

In this paper we have shown that the UHECR paradox can be explained in terms of a non-preferred frame effect of the laboratory frame which is moving with velocity $\approx 300 \mathrm{~km} / \mathrm{sec}$ with respect to the preferred one, assumed to be at rest with CMBR frame. Unlike some earlier efforts (the Coleman Glashow scheme for example) which consider LIV but assume that the physical kinematics is still Lorentzian, we propose to modify the transformation equation itself. Deformed LT are generally discussed in connection with test theories like that of Robertson [18] or Mansouri and Sexl [19] on which improved tests of SR are often based (see for example [20]). But they are not usually considered to represent a new physics that may provide a solution for the UHECR paradox. Some authors find it troublesome giving up the principle of relativity. In the so called "doubly special relativistic" [21,22] theories, the particle dispersion relation is modified but the introduction of an invariant length or energy scale in addition to the invariant velocity scale of SR, the "relativity of inertial frames" is still maintained. Such theories, often motivated by quantum-gravity considerations are interesting but are unable to resolve the UHECR paradox quantitatively at the moment. We here attempt to deform the relativistic kinematics using heuristic means. We do it first by identifying the objective contents of the relativity principle and then go in for modifying these contents minimally to obtain a new transformation that relates space-time of an arbitrary frame of reference with that of the universal rest frame of the cosmic substratum. The only phenomenological assumption regarding the speed of light in $\Sigma_{k}, c_{k}=c_{0}\left(1+\alpha \mathrm{u}_{\mathrm{k}}{ }^{2} / \mathrm{c}_{0}^{2}\right)^{1 / 2}$ (in contrast to the assumption, $c_{k}=$ $c_{0}$ in SR) for which $\quad c_{k}-c_{0}=\eta_{k} \approx 1.71 \times 10^{-22}$ in the laboratory frame $\left(u_{k} \approx 300 \mathrm{~km} / \mathrm{sec}\right)$, is the only speculative aspect that has been used to derive the new kinematics. Since the isotropy ingredient of the second relativity postulates has not been disturbed, Michelson-Morley type experiments cannot distinguish the proposed kinematics with that of the relativistic one. Also the limit on $\varepsilon$ given in Eq. (1) as 
a result of precision test becomes inconsequential, since the expected result in the present case would be zero. The recent improved test of time dilation in SR using laser spectroscopy sets a new limit of $2.2 \times$

$10^{-7}$ for deviation of time dilation factor [20]. This even does not match with the smallness of $\eta_{k}$ which is also the measure of this deviation according to the new kinematics. Hence the precision tests possibly will be unable to discern any deviation from SR in the near future, yet one may find an explanation of the cosmic ray paradox in the proposed deformed relativistic kinematics.

\section{Acknowledgment}

The author expresses sincere gratitude to Prof. S. K. Ghosal, Department of Physics, North Bengal University, India.

\section{References}

[1] S. Coleman, S.L. Glashow, Phys. Letts. B, 405 (1997) 249.

[2] S. Coleman, S. Glashow, Phys. Rev. D, 59 (1999) 116008.

[3] H. Sato, arXiv: astro-ph/0005218 v1, 2000.

[4] S .K. Lamoreaux, J.P. Jacobs, B.R. Heckel, F.J. Raab, E.N. Fortson, Phys. Rev. Lett., 57 (1986) 3125.

[5] D. J. Bird et.al, Astrophys. J., 441 (1995) 144.

[6] F. W. Stecker, S.T. Scully, arXiv: astro-ph/0412495 v3, 2005.

[7] K. Greisen, Phys. Rev. Lett., 16 (17) (1996) 748.

[8] G. Amelino-Camelia, Nature, 408 (2000) 661.

[9] G. Amelino-Camelia, T. Piran, Phys. Rev. D 64, (2001) 036005-1-12.

[10] G. Amelino-Camelia, Nature, 418 (2002) 34.

[11] T. Kifune, Astrophys. J., 518 (1999) L21-L24.

[12] S. Kovesi-Domoskos, G. Domoskos, arXiv: hep-ph/0501281 v1, 2005.

[13] George F.R. Ellis, Jean-Philippe Uzan, Am. J. Phys., 73 (2005) 240.

[14] S.K. Ghosal, D. Mukhopadhyay, P. Chakraborty, Eur. J. Phys., 15 (1994) 21.

[15] S.K. Ghosal, B. Raychaudhuri, A. K. Chowdhury, Minakshi Sarker, Found. Phys. Lett., 17 (2004) 457.

[16] C. Kacser, Introduction to the Special Theory of Relativity, (Edgewood Cliffs, NJ: Prentice-Hall), 1967.

[17] R. Baierlein, Am. J. Phys., 74 (2006) 193.

[18] H. P. Robertson, Rev. Mod. Phys., 21 (1949) 378.

[19] R. Mansouri, R. U. Sexl, Gen. Rel. Grav., 8 (1977) 497.

[20] G. Saathoff et.al, Phys. Rev. Lett., 91 (2003) 190403-1-4.

[21] G. Amelino-Camelia, Int. J. Mod. Phys. D, 11 (2002) 35.

[22] J. Magueijo, L. Smolin, Phys. Rev. Lett., 88 (2002) 190403-1-4. 